\title{
Uso de geotecnologias aplicadas em serviços de saúde: revisão Integrativa
}

\author{
Use of geotechnologies applied in health services: review integrative
}

Uso de geotecnologías aplicadas en servicios de salud: revisión integrativa

Kézia Porto Lima ${ }^{1 *}$, Dirceu Cavalcante Neves ${ }^{1}$, Natália Moreira Santos ${ }^{1}$, Sarah Lais Rocha ${ }^{1,2}$, Aline Santana Oliveira Valente ${ }^{3}$, Monica Cristina De Souza 4 .

\section{RESUMO}

Objetivo: Analisar o volume e características das produções científicas sobre uso de geotecnologias aplicadas em serviços de saúde. Métodos: Trata-se de uma revisão integrativa da literatura das bases de dados: Literatura Latino-Americano e do Caribe em Ciências da Saúde (LICAS), Scientific Electronic Library Online (SciELO) e Literature Analysis and Retrieval System Online (MEDLINE), com a utilização do programa Excel $\AA$, para criação de uma planilha com informações das publicações selecionadas para compor o estudo. Resultados: Identificou-se 20 artigos que respondiam aos nossos objetivos, destes apenas $30 \%$ eram nacionais e 70\% internacionais. O ano de maior publicação foi 2014 (30\%) e os enfermeiros e médicos realizaram $70 \%$ dos estudos. A revisão demonstrou que $60 \%$ das publicações foram de periódicos internacionais, sendo o Brasil e os E.U.A (30\%) responsáveis individualmente por $30 \%$ das publicações. A metodologia mais frequente foi pesquisa exploratória do tipo pesquisa bibliográfica (25\%); todas as publicações foram publicadas em periódicos diferentes. Conclusão: Foi constatado através desse estudo uma carência de produções carência de produções científicas sobre a temática em território nacional no campo da saúde. Tais tecnologias são ferramentas de excelência, evidenciando sua importância na promoção de melhores condições no campo da saúde, como: utilização consciente dos recursos, melhor acessibilidade dos usuários e redução de custos. Espera-se dos profissionais da saúde maior realização de estudos sobre a temática, bem como, desperte interesse para a construção de geotecnologias.

Palavras-chave: Mapeamento geográfico, Sistema de informação em saúde, Transporte de pacientes.

\section{ABSTRACT}

Objective: To analyze scientific production on the use of geotechnologies applied in health services. Methods: This is an integrative literature review of the databases: LILACS, SciELO and MEDLINE, using the Excel $\Theta$ program, to create a spreadsheet with information from selected publications to compose the study. Results: It was identified 20 articles that met our objectives, of which only $30 \%$ were national and $70 \%$ international. The year of greatest publication was 2014 (30\%) and nurses and doctors performed $70 \%$ of the studies. The review showed that $60 \%$ of publications were from international journals, with Brazil and the US $(30 \%)$ individually responsible for $30 \%$ of publications. The most frequent methodology was exploratory research of the bibliographic research type (25\%); All publications were published in different journals. Conclusion: Lack of scientific productions on the theme in the national territory in the health field. Such technologies are tools of excellence, highlighting their importance in promoting better conditions in the health field, such as: conscious use of resources, better user accessibility and cost reduction. Health professionals are expected to conduct further studies on the subject, as well as arouse interest for the construction of geotechnologies.

Keywords: Geographical mapping, Health information system, Patient transport.

\footnotetext{
${ }_{1}^{1}$ Faculdade Carajás, Marabá-Pará. *E-mail: keziaporto@hotmail.com

2 Universidade do Estado do Pará (UEPA) Marabá-Pará.

3 Universidade do Sul e Sudeste do Pará (UNIFESSPA), Marabá-Pará.

${ }^{4}$ Universidade do Estado do Rio De Janeiro (UERJ), Rio de Janeiro-Rio Janeiro.
} 


\section{RESUMEN}

Objetivo: analizar la producción científica sobre el uso de geotecnologías aplicadas en los servicios de salud. Métodos: Esta es una revisión integradora de la literatura de las bases de datos: LILACS, SciELO y MEDLINE, utilizando el programa Excel®, para crear una hoja de cálculo con información de publicaciones seleccionadas para componer el estudio. Resultados: Identificamos 20 artículos que cumplieron nuestros objetivos, de los cuales solo el 30\% eran nacionales y el 70\% internacionales. El año de mayor publicación fue 2014 (30\%) y las enfermeras y los médicos realizaron el 70\% de los estudios. La revisión mostró que el $60 \%$ de las publicaciones eran de revistas internacionales, con Brasil y los Estados Unidos (30\%) individualmente responsables del $30 \%$ de las publicaciones. La metodología más frecuente fue la investigación exploratoria del tipo de investigación bibliográfica (25\%); Todas las publicaciones fueron publicadas en diferentes revistas. Conclusión: Falta de producciones científicas sobre el tema en el territorio nacional en el campo de la salud. Dichas tecnologías son herramientas de excelencia, destacando su importancia en la promoción de mejores condiciones de salud, tales como: uso consciente de los recursos, mejor accesibilidad del usuario y reducción de costos. Se espera que los profesionales de la salud realicen más estudios sobre el tema, así como despertar interés por la construcción de geotecnologías.

Palabras clave: Mapeo geográfico, Sistema de información de salud, Transporte de pacientes.

\section{INTRODUÇÃO}

A evolução tecnológica tem impactado nos hábitos de vida da humanidade em todas esferas, científicas e sociais. O desenvolvimento de instrumentos, tem proporcionado melhorias significativas no cenário da saúde, contribuindo desde recurso diagnósticos as mais modernas terapias (CARROLL LN, et al., 2014; LONG LA, et al., 2018; COSTA PHA, et al., 2017).

O Geoprocessamento é um processo que localiza em um espaço geográfico eventos, selecionando características de dados de pessoas e os expõe espacialmente afim de considera-los como atributo daquele território. Esse processo resulta na representação gráfica simplificada das relações sociais e ambientais, as quais influenciam a ocorrência de determinada doenças ou condição de saúde (MACHARELLI CA, et al., 2013)

O geoprocessamento é constituído por um conjunto de tecnologias que coleta e processa informações espaciais para um determinado objetivo, que para ser alcançado, será realizado por sistemas programados para a aplicação desejada. Em grande parte, as bases de dados cartográficas digitais, são o resultado de planos de geoprocessamento, essas por sua vez, constituem apenas o ponto inicial da análise espacial do contexto de saúde. Essa ferramenta, só pode ser usada como meio de análise, se forem georreferenciados, associadas aos dados ambientais e socioeconômicos, e avaliada a sua distribuição espacial (MACHARELLI CA, et al., 2013)

As geotecnologias são técnicas matemáticas e computacionais de coleta, processamento e análises de informações a serem disponibilizadas com referências geográficas. Estas ferramentas são divididas em áreas: sensoriamento remoto, imagens de radar e perfis espectrais (PREZENTE WL, 2011). A criação de geotecnologias são instrumentos necessários na consolidação de geoprocessamento, tais recursos têm-se destacado no desenvolvimento de pesquisas, ferramentas como Sistema de Informação Geográfica (SIG), o georreferênciamento e a geolocalização ganharam notoriedade (KANG SY, et al., 2016; JUNIOR MS, et al., 2016).

O geomapeamento ou georreferênciamento é um processo no qual permite a identificação e avaliação de áreas geográficas. Ele estima dados descritivos sobre determinado local, como um endereço por exemplo, convertendo uma localização relacionada a um evento de saúde em uma representação visual gráfica. Tem sido utilizado na área da saúde, principalmente, na epidemiologia, mapeando doenças, surtos, entre outros (SILVEIRA IH, et al., 2017).

O SIG, possibilita pesquisadores visualizarem informações já referenciadas pelo geomapeamento. A aplicação desta ferramenta, dá-se através de software que permitirá descrever, analisar e prever padrões 
espaciais. Desta forma colabora significativamente para conhecimento epidemiológico de determinada área, favorecendo a implementação de programas de saúde em municípios ou regiões (SILVEIRA IH, et al., 2017) (ALMEIDA MCS, et al., 2014). Constitui-se assim, uma ferramenta importante para o diagnóstico situacional e planejamento de saúde pública (ALMEIDA MCS, et al., 2014).

Ainda de acordo com Ferraz, et al., (2015), tecnologias disponíveis e que permitem a um aprimoramento no desenvolvimento e avaliação de políticas públicas são as geotecnologias suportadas pelos Sistemas de Informações Geográficas (SIG). Elas possibilitam o processamento de grande quantidade de informações e permitem a integração de dados econômicos, sociais, ambientais e técnicos num contexto geográfico e temporal. Do ponto de vista prático, o assunto é novo e ainda está em desenvolvimento, portanto seu uso como ferramenta de controle ainda é muito incipiente.

A geolocalização é outra geotecnologia que facilita o entendimento dos dados, transformando esses em uma representação visual de forma gráfica e dinâmica. O posicionamento é baseado em informações e processamento de dados relacionados ao espaço geográfico por meio de um sistema de coordenadas, geralmente latitude e longitude, extraídas por técnica de geocoding (JUNIOR MS, et al., 2016).

Hoje em dia, essa estratégia pode ser observada pela representação visual desses dados por meio de sua visualização em serviços de mapas. Essa forma de visualização proporciona uma identificação mais clara de padrões em uma tal área geográfica apontada, exemplo de tais padrões podem ser tamanho de populações, a densidade de doenças e mudanças temporárias (JUNIOR MS, et al., 2016).

$\mathrm{Na}$ saúde pública, o uso de técnicas de geolocalização constitui-se como um passo de extrema importância para o desenvolver de um melhor atendimento à população, uma vez que essa estratégia auxilia no processo de compreensão de incidência de eventos, predição, tendência, simulações, planejamento e construção de estratégia no campo (OLIVEIRA EA, 2008; CABRAL APS, 2009; SKABA DA, 2009; BARCELLOS C, et al., 2008).

Bennett D (1991) evidencia que o uso das geotecnologias aplicado a questões de saúde coletiva, permitem o mapeamento de doenças, avaliação de riscos, planejamento de ações de saúde e a avaliação de redes de atenção como hospitais, unidades básicas de saúde, unidades de pronto atendimento, centros de referência de assistência social, entre outros.

Considerando essas informações, os objetivos foram analisar a produção científica sobre uso de geotecnologias aplicadas em serviços de saúde, visando identificar os principais cenários de utilização deste recurso, os países que mais implementaram estratégias de geomapeamento, bem como compreender os possíveis benefícios para as instituições e sociedade em geral.

\section{MÉTODOS}

Trata-se de um estudo do tipo revisão integrativa da literatura com finalidade de reunir e analisar resultados de pesquisa sobre o uso de geotecnologias aplicadas em serviços de saúde de maneira sistemática e ordenada, contribuindo para o aprofundamento do conhecimento do tema investigado (LIMA ACMACC, et al., 2016). Os seguintes Descritores em Ciências da Saúde: mapeamento geográfico; sistema de informação em saúde e transporte de pacientes.

A pesquisa incluiu artigos científicos disponíveis nas bases de dados: LILACS, SciELO e MEDLINE, e deviam ser de natureza humana e estar disponíveis na íntegra. Foram excluídos artigos que não respondiam ao objetivo, que não fossem de natureza humana, artigos não disponíveis na íntegra, dissertações e teses.

Após esta etapa, a amostra foi selecionada através da busca nas bases de dados, seguida da sumarização das informações extraídas dos artigos, avaliação dos estudos, interpretação e discussão dos resultados. A última etapa foi a construção da revisão.

O estudo utilizou uma planilha do programa Microsoft Excel® 2016 com informações sobre as publicações selecionadas para a composição deste estudo. Os resultados encontrados foram descritos por meio de quadros e tabelas, possibilitando ao leitor a avaliação da aplicabilidade da revisão integrativa, para atingir o objetivo desse método. 


\section{RESULTADOS E DISCUSSÃO}

A produção científica encontrada nas bases de dados foi submetida a leitura analítica e através desta foram aplicados os critérios de inclusão e exclusão, sendo a amostra final desta revisão integrativa constituída por 20 artigos científicos (Tabela 1).

Tabela 1 - Seleção dos artigos de pesquisa nas bases de dados LILACS, SciELO e MEDLINE, de acordo com os critérios de inclusão estabelecidos.

\begin{tabular}{|c|c|c|c|c|}
\hline Produção & LILACS & SciELO & MEDLINE & Total \\
\hline Produção encontrada & 15 & 3 & 25 & 43 \\
\hline $\begin{array}{c}\text { Não abordava a temática em } \\
\text { estudo }\end{array}$ & 4 & 1 & 1 & 6 \\
\hline Artigos repetidos & 3 & 1 & 6 & 10 \\
\hline Teses & 2 & 0 & 0 & 2 \\
\hline Artigos não disponíveis na íntegra & 0 & 0 & 5 & 5 \\
\hline Total & 6 & 1 & 13 & 20 \\
\hline
\end{tabular}

Fonte: LIMA KP, et al., 2019.

Evidenciou-se escassas produções científicas sobre georreferênciamento e a utilização de SIG em território nacional no cenário da saúde, somente $30 \%$ foram publicações brasileiras e $70 \%$ produções internacionais. O Brasil e os E.U.A foram os países que mais publicaram (30\%), seguidos pela África e Austrália que individualmente totalizaram $10 \%$ dos estudos identificados. Os países Argentina, Bélgica, China e Reino Unido individualmente representaram 5\% das publicações encontradas nesta revisão.

O ano com maior número de publicações foi 2014 (30\%) e a maioria (70\%) dos estudos sobre a-temática foi desenvolvida por enfermeiros e médicos.

A maioria dos artigos possuíam variadas metodologias, no entanto, destacou-se a pesquisa exploratória do tipo pesquisa bibliográfica (25\%). Em relação aos periódicos, dos 20 artigos todos foram publicados em periódicos diferentes. Representação da síntese da produção científica analisada. (Quadro 1). 
Quadro 1 - Representação da síntese da produção científica analisada.

\begin{tabular}{|c|c|c|c|c|}
\hline Autores & Ano & $\begin{array}{l}\text { Base de } \\
\text { dados }\end{array}$ & Objetivo & Resultados \\
\hline $\begin{array}{l}\text { Junior MS, } \\
\text { et al. }\end{array}$ & 2016 & LILACS & $\begin{array}{l}\text { Aplicar técnicas de geolocalização a partir } \\
\text { do rastreamento em tempo real. }\end{array}$ & $\begin{array}{c}\text { Implantação de dashboard maximizando a eficiência na logística } \\
\text { dos atendimentos e racionalização dos custos. }\end{array}$ \\
\hline $\begin{array}{l}\text { Zhang GX, } \\
\text { et al. }\end{array}$ & 2017 & LILACS & $\begin{array}{l}\text { Caracterizar o espaço geográfico da } \\
\text { distribuição de incidentes de trauma e } \\
\text { analisar o nível de instalações de saúde de } \\
\text { destino dos pacientes. }\end{array}$ & $\begin{array}{l}\text { Os incidentes ocorreram predominantemente na parte ocidental } \\
\text { de Shenzhen. Os destinos foram hospitais de ensino, regional, } \\
\text { comunitário e privado. }\end{array}$ \\
\hline $\begin{array}{l}\text { Macharelli } \\
\text { CA, et al. }\end{array}$ & 2013 & LILCAS & $\begin{array}{l}\text { Analisar o comportamento espacial da } \\
\text { ocorrência de casos de tracoma para } \\
\text { definir áreas prioritárias. }\end{array}$ & $\begin{array}{l}\text { Foi possível realizar a distribuição e mapear: a densidade da } \\
\text { ocorrência de tracoma de acordo com a renda familiar na área } \\
\text { urbana; e levando em consideração a escolaridade do chefe de } \\
\text { família. }\end{array}$ \\
\hline $\begin{array}{l}\text { Costa PHA, } \\
\text { et al. }\end{array}$ & 2017 & LILACS & $\begin{array}{l}\text { Apresentar georreferenciamento e análise } \\
\text { de serviços baseado em tecnologias e } \\
\text { processos de acesso livre. }\end{array}$ & $\begin{array}{l}\text { Foram identificados e georreferenciados } 187 \text { serviços, e suas } \\
\text { interações caracterizadas pela análise de redes sociais. }\end{array}$ \\
\hline $\begin{array}{l}\text { Almeida } \\
\text { MCS, et al. }\end{array}$ & 2014 & LILACS & $\begin{array}{l}\text { Identificar padrões espaciais da } \\
\text { distribuição da mortalidade por doença de } \\
\text { Alzheimer. }\end{array}$ & $\begin{array}{l}4 \text { municípios apresentaram altas taxas óbitos por Alzheimer. } 42 \\
\text { municípios merecem intervenção, identificou-se alta densidade } \\
\text { de óbitos na região noroeste do estado. }\end{array}$ \\
\hline $\begin{array}{l}\text { Romano } \mathrm{H} \text {, } \\
\text { et al. }\end{array}$ & 2017 & LILACS & $\begin{array}{l}\text { Fornecer sistema de dados úteis para } \\
\text { registrar e detectar associações de câncer } \\
\text { e possíveis locais de risco. }\end{array}$ & $\begin{array}{c}\text { Desenvolvido e implementado sistema de georreferênciamento } \\
\text { ao problema de câncer ou outras doenças. Visualização de } \\
\text { dados ambientais e localização de fontes potencialmente } \\
\text { oncogênicas. }\end{array}$ \\
\hline $\begin{array}{l}\text { Silveira } \mathrm{IH}, \\
\quad \text { et al. }\end{array}$ & 2017 & SCIELO & $\begin{array}{l}\text { Aplicação de um procedimento de baixo } \\
\text { custo, baseado em software livre, para o } \\
\text { georreferenciamento de dados do Sistema } \\
\text { de Informações sobre Mortalidade. }\end{array}$ & $\begin{array}{c}91,6 \% \text { dos endereços submetidos ao procedimento foram } \\
\text { georreferenciados. }\end{array}$ \\
\hline
\end{tabular}

REAS/EJCH | Vol.12(6) | e3072 | DOI: https://doi.org/10.25248/reas.e3072.2020 Página 5 de 11 


\begin{tabular}{|c|c|c|c|c|}
\hline $\begin{array}{l}\text { Hsia R.Y.; } \\
\text { Shen Y.C. }\end{array}$ & 2016 & $\begin{array}{l}\text { MEDLIN } \\
\qquad \mathrm{E}\end{array}$ & $\begin{array}{l}\text { Determinar como o acesso à intervenção } \\
\text { coronariana percutânea (PIC) é distribuído } \\
\text { demograficamente. }\end{array}$ & $\begin{array}{l}\text { Tempo médio pré-hospitalar para o centro } \mathrm{PCI} \text { mais próximo é de } \\
33 \text { minutos. } 84 \% \text { vivem cerca de uma hora de um centro de } \mathrm{PCl} \text {. } \\
\text { Maior probabilidade de acesso inoportuno, em baixa renda, rural } \\
\text { e comunidades altamente hispânicas. }\end{array}$ \\
\hline $\begin{array}{l}\text { Klimas J, et } \\
\text { al. }\end{array}$ & 2014 & $\begin{array}{l}\text { MEDLIN } \\
\qquad \mathrm{E}\end{array}$ & $\begin{array}{l}\text { Identificar incidência de óbitos por } \\
\text { overdose nos serviços de ambulância e } \\
\text { sua relação com o distanciamento urbano } \\
\text { e presença de serviços de dependência. }\end{array}$ & $\begin{array}{l}13 \text { óbitos por overdose; Superdoses mais prováveis em áreas } \\
\text { afluentes cerca de } 1000 \text { m de serviços de dependência. } \\
\text { Superdosagens residenciais em áreas mais carentes. Overdose } \\
\text { em ruas habitualmente no centro da cidade. }\end{array}$ \\
\hline $\begin{array}{l}\text { Kang SY, et } \\
\text { al. }\end{array}$ & 2016 & $\begin{array}{l}\text { MEDLIN } \\
\qquad \mathrm{E}\end{array}$ & $\begin{array}{c}\text { Fornecer compreensão básica dos } \\
\text { principais conceitos envolvidos nos } \\
\text { métodos de mapeamento da doença por } \\
\text { abordagem bayesiana para dados de área. }\end{array}$ & $\begin{array}{l}\text { Métodos de mapeamento de doenças, explicando os conceitos, } \\
\text { termos técnicos e ilustrando a utilidade do mapeamento de } \\
\text { doenças para a pesquisa epidemiológica. }\end{array}$ \\
\hline $\begin{array}{l}\text { MunyaneZA } \\
\text { F, et al. }\end{array}$ & 2014 & $\begin{array}{l}\text { MEDLIN } \\
\qquad \mathrm{E}\end{array}$ & $\begin{array}{l}\text { Descrever o uso supervisionado de } \\
\text { agentes comunitários de saúde para } \\
\text { mapear aldeias e a variação em nível de } \\
\text { aldeia na disponibilidade de água potável. }\end{array}$ & $\begin{array}{c}\text { Mapeamento de } 573 \text { aldeias do distrito usando } 12 \text { dispositivos } \\
\text { GPS compartilhados. Mapas setoriais foram produzidos e } \\
\text { distribuídos a autoridades locais. }\end{array}$ \\
\hline $\begin{array}{l}\text { Almeida } \\
\text { TSO, et al. }\end{array}$ & 2018 & $\begin{array}{l}\text { MEDLIN } \\
\qquad \mathrm{E}\end{array}$ & $\begin{array}{l}\text { Mapear as áreas de incidência de } \\
\text { tentativas de suicídio. }\end{array}$ & $\begin{array}{l}446 \text { tentativas de suicídios georreferenciados, incidência de } 120 \\
\text { casos a cada } 100.000 \text { habitantes. }\end{array}$ \\
\hline $\begin{array}{l}\text { CotteelS C, } \\
\text { et al. }\end{array}$ & 2012 & $\begin{array}{l}\text { MEDLIN } \\
\qquad \mathrm{E}\end{array}$ & $\begin{array}{l}\text { Mapear as localizações atuais dos centros } \\
\text { de radioterapia em todo o país e avaliar as } \\
\text { disparidades espaciais observadas. }\end{array}$ & $\begin{array}{l}\text { Notou-se que a situação atual é, em média, aceitável em termos } \\
\text { de acessibilidade dos usuários aos serviços. }\end{array}$ \\
\hline $\begin{array}{l}\text { GoldberG } \\
\text { DW.; } \\
\text { Jacquez } \\
\text { GM. }\end{array}$ & 2012 & $\begin{array}{l}\text { MEDLIN } \\
\qquad \mathrm{E}\end{array}$ & $\begin{array}{l}\text { Analisar os resumos das edições especiais } \\
\text { selecionados e submetidos. }\end{array}$ & $\begin{array}{c}\text { Cada trabalho revela que as geotecnologias são aplicáveis em } \\
\text { qualquer trabalho e estudo, que problemas de precisão espacial, } \\
\text { viés geográfico e geocodificação são independes da localização } \\
\text { e suas limitações de acordo com autor. }\end{array}$ \\
\hline $\begin{array}{l}\text { FreestoNE } \\
\mathrm{D}, \text { et al. }\end{array}$ & 2012 & $\begin{array}{l}\text { MEDLIN } \\
\qquad \mathrm{E}\end{array}$ & $\begin{array}{l}\text { Estabelecer os fatores que afetaram a } \\
\text { qualidade dos dados geocodificados e a } \\
\text { extensão desse impacto. }\end{array}$ & $\begin{array}{c}\text { Identificou-se que a documentação de origem, a limpeza de } \\
\text { dados e as configurações de software como fatores-chave que } \\
\text { afetam a qualidade dos dados. }\end{array}$ \\
\hline
\end{tabular}

REAS/EJCH | Vol.12(6) | e3072 | DOI: https://doi.org/10.25248/reas.e3072.2020 Página 6 de 11 


\begin{tabular}{|c|c|c|c|c|}
\hline $\begin{array}{l}\text { Rereddy SK, } \\
\text { et al. }\end{array}$ & 2015 & $\begin{array}{l}\text { MEDLIN } \\
\text { E }\end{array}$ & $\begin{array}{l}\text { Explorar distribuição dos casos de câncer } \\
\text { de } 2010 \text { a 2012. Identificar tendências } \\
\text { geográficas na prevalência da doença em } \\
\text { comparação com a distribuição de } \\
\text { prestadores de cuidados de saúde orais } \\
\text { por CEP de suas localizações. }\end{array}$ & $\begin{array}{c}\text { Identificados } 53 \text { casos, distribuídos por } 36 \text { códigos postais. SIG } \\
\text { demonstrou desajuste espacial: aumento da prevalência da } \\
\text { doença e escassez de provedores nos códigos postais e } \\
\text { diminuição da prevalência da doença e maior presença dos } \\
\text { provedores nos códigos postais majoritários. }\end{array}$ \\
\hline $\begin{array}{l}\text { Richards M, } \\
\text { et al. }\end{array}$ & 2014 & $\begin{array}{c}\text { MEDLIN } \\
\text { E }\end{array}$ & $\begin{array}{l}\text { Determinar tempo de transporte materno } \\
\text { terrestre de hospitais comunitários para } \\
\text { hospital mais próximo nível III. }\end{array}$ & $\begin{array}{l}\text { As proporções dos hospitais nível I e ll eram de uma viagem de } \\
30 \text { minutos de centro neonatal nível III foram de } 19,8 \text { e } 47,3 \% \text {, e } \\
52,2 \% \text { e } 69,8 \% \text { estavam dentro do tempo de } 60 \text { minutos. }\end{array}$ \\
\hline Neal S, et al. & 2016 & $\begin{array}{l}\text { MEDLIN } \\
\text { E }\end{array}$ & $\begin{array}{l}\text { Avaliar abrangentemente a distribuição } \\
\text { geográfica dos primeiros filhos de mães } \\
\text { adolescentes usando dados demográficos } \\
\text { e domésticos descritivos, de análise } \\
\text { espacial e de modelagem espacial. }\end{array}$ & $\begin{array}{l}\text { Acentuada heterogeneidade geográfica entre os primeiros } \\
\text { nascimentos de gestantes adolescentes. Muitas áreas de alta } \\
\text { prevalência podem estar ligadas à pobreza subjacente. }\end{array}$ \\
\hline $\begin{array}{l}\text { Carroll LN, } \\
\text { et al. }\end{array}$ & 2014 & $\begin{array}{l}\text { MEDLIN } \\
\text { E }\end{array}$ & $\begin{array}{l}\text { Identificar necessidades e preferências de } \\
\text { usuários de saúde pública para } \\
\text { ferramentas de visualização de } \\
\text { informações sobre doenças infecciosas; }\end{array}$ & $\begin{array}{l}\text { Diversas necessidades, preferências e usos dessas ferramentas. } \\
\text { Estudos identificaram preocupações do compartilhamento de } \\
\text { dados, confidencialidade e qualidade. Instrumentos oferecem } \\
\text { vários recursos e funções permitindo que os usuários explorem, } \\
\text { analisem e visualizem dados. }\end{array}$ \\
\hline $\begin{array}{c}\text { Delmelle EM } \\
\text { et al. }\end{array}$ & 2013 & $\begin{array}{c}\text { MEDLIN } \\
\text { E }\end{array}$ & $\begin{array}{l}\text { Estimar o tempo de viagem em relação a } \\
\text { distância para acesso aos cuidados } \\
\text { médicos utilizando SIG. }\end{array}$ & $\begin{array}{l}99,7 \% \text { dos endereços residenciais maternos foram } \\
\text { geocodificados. Crianças com espinha bífida de áreas rurais na } \\
\text { Flórida experimentaram tempos de viagem quase o dobro em } \\
\text { comparação com aqueles que vivem em áreas urbanas. } \\
\text { Agrupados em condados os tempos de viagem de rede } \\
\text { unidirecional exibiram auto correlação espacial estatisticamente } \\
\text { significativa, indicando que famílias que vivem em alguns grupos } \\
\text { de condados possuem tempos de viagem maiores em } \\
\text { comparação com as famílias que moram em outras áreas. }\end{array}$ \\
\hline
\end{tabular}

Fonte: Lima KP, et al., 2019.

REAS/EJCH | Vol.12(6) | e3072 | DOI: https://doi.org/10.25248/reas.e3072.2020 Página 7 de 11 
Técnicas de georreferênciamento podem ser aplicados na área da saúde como métodos potencialmente inovadores na pesquisa, gestão, atuação profissional, bem como para os usuários da rede (CARROL LN, et al., 2013) (SILVEIRA IH, et al., 2017) (COSTA PHA, et al., 2017). Um compilado de artigos que tratam desta temática pontuando os métodos e os dados secundários obtidos nesta revisão de forma cronológica, demonstra o modo como essas inovações tecnológicas tem sido aplicada no cenário da saúde internacional e nacional.

Estudo realizado nos E.U.A durante o período de 1998 - 2008 identificou 668 lactentes portadores de espinha bífida. Por meio da geocodificação foi mapeado a localização desses bebes e calculado o tempo gasto até os serviços de saúde mais próximo de suas residências. O estudo aponta para uma maior densidade hospitalar em áreas urbanas o que aumenta o tempo de viagem até os centros de saúde, pois grande parte dessas crianças residem em áreas rurais. Esses dados podem auxiliar aos profissionais e pesquisadores em saúde a elaborarem estratégias que melhorem a oferta desses serviços (DELMELLE EM et al., 2013).

Em 2008 mapeou-se informações sobre gravidez precoce em adolescentes de países da África. Foram utilizados dados demográficos e um questionário socioeconômico usando métodos descritivos de análise e de modelagem espacial. As análises foram destinadas a jovens entre 16 e 19 anos de idade e verificou-se acentuada heterogeneidade geográfica na gravidez precoce, principalmente nos grupos de menor idade. As áreas de maior incidência foram associadas a pobreza subjacente. As conclusões da pesquisa fornecem dados que viabilizam elaboração de políticas que concentrem recursos onde são mais necessários para amenizar a situação atual (NEAL S, et al., 2016).

Em Dublin na Irlanda, foram analisados no período de doze meses os serviços de ambulâncias com intuito de verificar a incidência de overdoses por opiáceos no ano de 2011. As informações utilizadas foram coletadas dos relatórios de atendimento ao paciente que tornaram possível o mapeamento dos locais de maior ocorrência de overdose. Saber onde e como acontecem as overdoses é importante para o treinamento preventivo das equipes de socorro e atuação de serviços de educação em saúde nesta população (KLIMAS J, et al., 2014).

O distrito de Burera em Uganda é uma região montanhosa com topografia que varia entre $1.728 \mathrm{~m}$ para $4.098 \mathrm{~m}$ de altitude acima do nível do mar. Esta região foi objeto de um estudo em 2011 que aproveitou o serviço de agentes comunitários de saúde para mapear de forma rápida e barata aldeias de difícil acesso (MUNYANEZA F, et al., 2014).

Uma equipe de profissionais foi treinada para manusear GPS e recolherem coordenadas geográficas das aldeias, os dados foram georreferenciados com a utilização do software ArcMap 10, ao longo de 12 meses foram mapeadas 573 aldeias e os mapas produzidos foram distribuídos para as autoridades, pois forneciam dados importantes da identificação de variabilidade na saúde e outros serviços locais orientando para as possíveis intervenções (MUNYANEZA F, et al., 2014).

Estudo realizado na Austrália em 2012 baseou-se na coleta de informações do sistema nacional de informação de médicos legistas, que funciona como ferramenta de vigilância de lesões. A pesquisa contou com geocodificação das informações relativas à morte. Este processo visou enriquecer o valor dos dados contidos no sistema, desenvolver e manter um serviço de informação de alta qualidade para médicos legistas, gestores públicos e pesquisadores para beneficiar a população Australiana (FREESTONE D, et al., 2012).

Em 2012, na Bélgica, foi proposto um modelo que mapeou os centros de tratamentos radiológicos verificando as distâncias entre estes centros e os usuários da rede de atendimento, especificamente pacientes em tratamento de câncer. Este estudo verificou que a população usuária desses serviços de radiologia, estavam distantes dos seus respectivos locais de tratamento. Esta pesquisa foi considerada um importante ponto de partida para compreensão geopolítica dos serviços de saúde neste país (COTTEELS C, et al., 2012).

Uma revisão bibliográfica realizada nos E.U.A no ano do 2013 analisou 88 artigos que identificou as principais ferramentas de visualização de doenças infecciosas. Gráficos, mapas e relatórios compõem importantes ferramentas para extração de dados relacionados a saúde, todavia, é necessário incentivar a 
criação de mais ferramentas que proporcionam o compartilhamento de informações entre os usuários dos serviços de saúde e o poder público, favorecendo a criação de um banco de dados robusto que auxilie na identificação de padrões de doenças infecciosas e dê suporte aos profissionais de saúde e na tomada de decisões dos gestores públicos (CARROL LN, et al., 2013).

Recentemente um estudo chinês mapeou a distribuição geográfica dos incidentes envolvendo vítimas com trauma e analisou a estrutura dos hospitais de destino desses pacientes. Zhang e colaboradores (2017) evidenciaram que a política de levar o paciente para hospitais mais próximos do local do evento traumático é praticada pelos serviços de emergência, mesmo não tendo garantia de que este seja equipado ou disponha de pessoal especializado para esse tipo de ocorrência (ZHANG GX, et al., 2017).

Tal decisão, pode impactar no tratamento e recuperação destes pacientes, conclui-se que a partir dos dados coletados e georreferenciados, que há necessidade de criação de um protocolo de triagem préhospitalar, afim de organizar o fluxo de pacientes para esses centros especializados (ZHANG GX, et al., 2017).

Nos EUA foi realizado uma pesquisa geográfica dos hospitais que oferecem serviços de maternidade em 2017. Neste estudo avaliou-se o tempo de transporte materno terrestre de hospitais níveis I e II para centros neonatais nível III, relatou-se também o tempo de condução até a maternidade de gestantes que poderiam necessitar de cuidados especializados para si ou para o recém-nascido. Verificou se que $50 \%$ dos trajetos aos hospitais nível I e um terço dos trajetos dos hospitais nível II, foram necessários mais de 60 minutos para chegar ao centro neonatal nível III mais próximo (RICHARDS M, et al., 2014).

O mapeamento de serviços de saúde, ajudou a ressaltar importância dos hospitais nível I e II e seus meios de transporte estratégicos em estados com menor densidade populacional (RICHARDS M, et al., 2014).

Em 2015, na cidade de Atlanta nos EUA buscou-se por meio do SIG informações de registros médicos sobre câncer de cabeça e pescoço em áreas de escassez de provedores de saúde. Os dados obtidos forneceram uma imagem da carga da doença na região e com isso gerou informações a serem incorporadas a um banco de dados para consultas futuras (REREDDY SK, et al., 2014). Semelhantemente, um estudo na Austrália baseou-se no método Bayesiana de modelagem espacial para mapear casos de câncer (KANG SY, et al., 2016).

Em 2017, foi realizado na cidade Bahia Blanca, Argentina, um sistema de georreferênciamento sobre a incidência dos casos de câncer, a qual contou com um grupo de trabalho multidisciplinar nas áreas de saúde, computação, geografia e estatística (ROMANO H, et al., 2017).

Este sistema permitiu determinar qual a incidência dos casos da doença curto, médio e longo prazo. Como resultado da pesquisa obteve-se uma ferramenta com potencial para extração de dados estatísticos que estabelecem combinações com tipos de câncer, idade, sexo, evolução e mortalidade dos pacientes além de suas localizações em tempo real (ROMANO H, et al., 2017).

Um levantamento realizado nos E.U.A baseou-se na coleta de dados de 1.738 centros de intervenção coronária percutânea através do cruzamento de 32.370 códigos postais representando um total de 306 milhões de americanos. Através de georreferenciamento dos endereços e a criação de um mapa temático a pesquisa demonstrou que $52 \%$ tem acesso prematuro aos centros de $\mathrm{PCl}$ enquanto $48 \%$ não dispõem de um acesso adequado (HSIA RY e SHEN YC, 2016).

No Brasil, um sistema de informação sobre mortalidade foi realizado no município do Rio de Janeiro-RJ no período 2010-2012, que consistiu no georreferênciamento de 26.081 endereços de óbitos por doenças isquêmicas do coração e cerebrovasculares. Desses endereços, $75 \%$ foram georreferenciados automaticamente pelo software, os endereços remanescentes foram submetidos a intervenção manual (SILVEIRA IH, et al., 2017).

Os dados foram obtidos no Sistema de Informação de Mortalidade (SIM). A pesquisa baseou-se em três etapas: a padronização dos endereços, o georreferênciamento por meio do Google Maps e a intervenção manual. O georreferênciamento neste estudo teve alta proporção de acertos, apesar das dificuldades relacionadas à qualidade dos dados e as condições da infraestrutura urbana. Reconhecer a distribuição 
espacial de uma doença é importante para identificar locais de maior incidência e assim atuar na conscientização da população para prevenção (SILVEIRA IH, et al., 2017). Na cidade de Bauru-SP foi realizada uma análise da distribuição espacial dos casos de tracoma. Identificando 66 casos em uma amostragem aleatória envolvendo 12 escolas municipais. O georreferênciamento utilizou GPS que após a coleta de dados gerou informações sobre a localização da população atingida (MACHARELLI CA, et al., 2013).

O mapeamento foi disponibilizado e utilizado pelo IBGE no Censo demográfico. Os dados obtidos forneceram informações sobre a população afetada que coincidiu com as áreas de maior desigualdade social na cidade de Bauru. As microáreas identificadas, foram aquelas que devem ser priorizadas na distribuição dos recursos em saúde. Existe ainda a possibilidade de usar os casos de tracoma identificados como indicador de desempenho de saúde (MACHARELLI CA, et al., 2013).

No estado de São Paulo foi desenvolvido um estudo em 2014 acerca da distribuição espacial das taxas de mortalidade por doença de Alzheimer. Foram utilizadas informações do SIM no DATASUS. Através dos dados obtidos foram mapeados os municípios com as maiores taxas de óbito devido a doença de Alzheimer e a identificação dos locais que tem prioridade de intervenção para a diminuição das taxas de óbitos por essa doença (ALMEIDA MCS, et al., 2014). Em 2016, na cidade de Maceió-AL realizou-se um estudo que consistiu no rastreamento em tempo real de quatro ambulâncias (identificadas como USA10, USA12, USB06 e USB57). Baseou-se numa arquitetura de software que monitorou os dados enviados via GPS que eram salvos no banco de dados do servidor Traccar, sendo exibidos em um mapa online, que apresentavam rastreamento dos veículos com dados de velocidade do veículo, hora, data e identificador da ambulância (JUNIOR MS, et al., 2016).

As coordenadas então foram processadas e plotadas nos mapas em tempo real, o que permitiu o acompanhamento do deslocamento dos veículos e a possibilidade de traçar e analisar as rotas. Os dados geolocalizados das ambulâncias (rastreamento via GPS) foram agregados em um painel único de visualização em camadas. Observou-se a integração entre os gráficos gerados pelo perfil das vítimas e ocorrência e o rastreamento das ambulâncias no mapa (JUNIOR MS, et al., 2016). Em 2017, georreferênciamento foi utilizado em Juiz de Fora- MG para mapear as redes de atenção ao usuário de drogas com a utilização da-plataforma Google Earth e Google Fusion Tables para georreferenciar os serviços a partir de seus endereços, além disso foram adicionadas outras informações relevantes como o tipo de serviço, público-alvo, modelo de tratamento e etc (COSTA PHA, et al., 2017).

Um estudo recente, em Campina Grande -PB relatou que o geoprocessamento ajudou a identificar dados sobre casos confirmados de tentativas de suicídio. A pesquisa foi desenvolvida com base em casos de autointoxicação por pesticidas, remédios e substâncias de limpeza em que 446 casos foram selecionados, apontando uma incidência de 120 ocorrências por 100.000 habitantes. Os endereços das vítimas foram cadastrados e identificados no site da agência postal e depois de georreferenciados, rastreados por meio do Google Earth (ALMEIDA TSO, et al., 2018).

\section{CONCLUSÃO}

O uso de tais tecnologias quando aplicadas na área da saúde, constituem-se uma ferramenta de grande importância, mapeando distribuição de serviços de saúde, distância e o tempo percorrido paciente-serviço, distribuição parcial de doenças ou, até mesmo, acidentes. O georreferênciamento também é eficiente na elaboração de produtos, como no desenvolvimento de protocolos, painéis de controle ou bancos de dados, permitindo uma visão geral das condições ambientais e populacionais, fornecendo mapas e por fim, na criação de produtos que permite a observação da distribuição espacial de exposições a riscos e problemas de saúde. Desta forma, através desta pesquisa, espera-se que os profissionais da saúde tenham mais interesse sobre a temática e realizem novos estudos sobre o uso de geotecnologias aplicadas em serviços de saúde, bem como criem geotecnologias aplicadas ao cenário da saúde brasileira.

\section{REFERÊNCIAS}

1. ALMEIDA MCS, et al. Spatial distribution of deaths due to Alzheimer's disease in the state of São Paulo, Brazil. Sao Paulo Med. J., 2014; 132(4): 199-204. 
2. ALMEIDA TSO, et al. Suicide attempts: epidemiologic trends towards geoprocessing. Ciênc. Saúde Coletiva, 2018; 23(4), 1183-1192.

3. BARCELLOS, C. et al. Georreferenciamento de dados de saúde na escala submunicipal: algumas experiências. Epidemiol. Serv. Saúde, 59-70, Brasília, 2008.

4. BENNETT D. Explanation in medical geography.Evidence and epistemology. Social Science and medicine, 1991; (33): 339-346.

5. CABRAL, APS. Serviço de atendimento móvel de urgência: um observatório dos acidentes de transporte terrestre, Recife, 2009.

6. CARROLL LN, et al. Visualization and analytics tools for infectious disease epidemiology: A systematic review. Journal of Biomedical Informatics, 2014; 51: 287-298.

7. COSTA PHA, et al. Tecnologias de acesso livre para georreferenciamento e análise de sistemas e redes de atenção aos usuários de drogas. Revista Eletrônica de Comunicação, Informação e Inovação em Saúde, 2017 ; 11 (4): 1 -18.

8. COTTEELS C, et al. Localisation des centres de radiothérapie: une analyse géographique exploratoire pour la Belgique. Cancer/Radiothérapie, 2012; 16: 604 -612.

9. DELMELLE EM, et al. Modeling Travel Impedance to Medical Care for Children with Birth Defects Using Geographic Information Systems. Birth Defects Research (Part A), 2013; 97:673-684.

10. FERRAZ CAM, et al. O uso de geotecnologias como uma nova ferramenta para o controle externo. Rev do TCU. 2015;(133).

11. FREESTONE D, et al. Geocoding coronial data: tools and techniques to improve data quality. Health Inf Manag, 2012; 41 (3): 4-12.

12. GOLDBERG DW, JACQUEZ GM. Advances in geocoding for the health sciences. Spatial and Spatio-temporal Epidemiology, 2012; 3:1-5.

13. HSIA RY.; SHEN YC. Percutaneous Coronary Intervention in the United States: Risk Factors for Untimely Access. Health Serv Res, v.51, n.2, p.592-609, 2016. doi: 10.1111/1475-6773.12335.

14. JUNIOR MS, et al. Geolocalização aplicada aos atendimentos do samu 192 a partir do rastreamento em tempo real das Ambulâncias. XV Congresso Brasileiro de Informática em Saúde, 2016.

15. KANG SY, et al. Making the most of spatial information in health: a tutorial in Bayesian disease mapping for areal data. Geospatial Saúde, 2016; 11: 428: 190-198.

16. KLIMAS J, et al. Urban overdose hotspots: a 12-month prospective study in Dublin ambulance services. American Journal of Emergency Medicine, 2014; 32: 1168-1173.

17. LIMA ACMACC, et al. Nursing diagnoses in patientswith cerebral vascular accident: anintegrative review. Rev. Bras. Enferm, 2016; 69 (4): 738-45.

18. LONG LA, et al. Digital Technologies for Health Workforce Development in Low- and Middle-Income Countries: A Scoping Review. Global Health: Science and Practice, 2018; 6:41-48.

19. MACHARELLI CA, et al. Spatial distribution of trachoma cases in the City of Bauru, State of São Paulo, Brazil, detected in 2006: defining key areas for improvement of health resources. Rev. Soc. Bras. Med. Trop, 2013; 46(2): 190-195.

20. MUNYANEZA F, et al. Leveraging community health worker system to map a mountainous rural district in low resource setting: a low-cost approach to expand use of geographic information systems for public health. International Journal of Health Geographics, 2014; 13(49):1-8.

21. NEAL S, et al. Mapping adolescent first births within three east African countries using data from Demographic and Health Surveys: exploring geospatial methods to inform policy. Reproductive Health, 2016; 13(1): 1-29.

22. OlIVEIRA, EA de. Otimização da localização das bases de operação do Serviço de Atendimento Móvel de Urgência: SAMU/BH através do uso de técnicas de Geoprocessamento. Caminhos de Geografia, v.9, n. $26,2008$.

23. PREZENTE, WL. O uso das Geotecnologias no ensino da Geografia. Anais do I Seminário Internacional de Espaço de Fronteira, 2011.

24. REREDDY SK, et al. Dying to be Screened: Exploring the Unequal Burden of Head and Neck Cancer in Health Provider Shortage Areas. Journal of Cancer Education, 2014; 30(3):490-496.

25. RICHARDS M, et al. Geographical Information Systems for Mapping Maternal Ground Transport to Level III Care Neonatal Centers. American Journal of Perinatology, 2013; 31 (4): 287-292.

26. ROMANO H, et al. - Generación y prueba de un sistema de georreferenciamiento de cáncer en la ciudad de bahía blanca - Development and testing of a cancer georeferencing system in the city of Bahía Blanca. Rev. Asoc. Med. Bahía Blanca, 2017; 27(1): 9-18.

27. SILVEIRA IH, et al. Utilização do Google Maps para o georreferenciamento de dados do Sistema de Informações sobre Mortalidade no município do Rio de Janeiro, 2010-2012*. Epidemiol. Serv. Saúde, 2017; 26(4):881-886.

28. SKABA, DA. Metodologias de Geocodificação dos Dados da Saúde, Rio de Janeiro - RJ, 2009.

29. ZHANG GX, et al. An Exploratory Analysis of the Geographical Distribution of Trauma Incidents in Shenzhen, China. World J Surg, 2017; 41(9):2207-2214. 